Gefässchirurgie 2011 · 16:453-453 DOI 10.1007/s00772-011-0894-2

Online publiziert: 2. Oktober 2011

(c) Springer-Verlag 2011

T. Kölbel · E.S. Debus

Klinik und Poliklinik für Gefäßmedizin, Universitäres Herzzentrum, Universitätsklinikum Hamburg-Eppendorf, Hamburg

\title{
Kontrastmittelsicherheit: Die andere Seite der Medaille
}

bei nierengesunden Patienten handelt es sich um eine häufige iatrogen verursachte Gesundheitsstörung.

Auf die Kontrastmittelgabe kann dennoch in der Medizin nicht verzichtet werden. Besonders die Angiographie mit jodhaltigem Kontrastmittel gilt in vielen Bereichen als Goldstandard in der diagnostischen Darstellung der Gefäßanatomie. Die Herausforderung für jeden Gefäßmediziner liegt daher darin, die Risiken der Kontrastmittelgabe gegen den diagnostischen und therapeutischen Nutzen bei jedem Patienten individuell abzuwägen, Risikofaktoren rechtzeitig zu erkennen und zu minimieren sowie Alternativverfahren zu kennen und gezielt einzusetzen.

Die Geschichte der Kontrastmittel ist dabei fast so alt wie die Geschichte der Röntgenstrahlen selbst. Bereits 1914 wurde Kohlendioxid als Kontrastmittel verwendet, und seit 1924 werden jodhaltige Kontrastmittel intravenös eingesetzt. Jodhaltige Kontrastmittel haben seit dieser Zeit eine Reihe von innovativen Änderungen erfahren. Die heute üblichen wasserlöslichen nichtionischen Kontrastmittel haben eine deutlich geringere Nebenwirkungsrate und sind sicherer in der Anwendung geworden. Zusätzlich haben Optimierungen in der Durchleuchtungstechnik und Angiographie dazu beigetragen, dass geringere Kontrastmittelmengen erforderlich sind. Da jedoch in den vergangenen 30 Jahren bildgebende Untersuchungsverfahren, die mithilfe eines Kontrastmittels durchgeführt werden, stark zugenommen haben, hat auch die Anzahl der Kontrastmittelnebenwirkungen, wie die der kontrastmittelinduzierten Nephropathie, zugenommen. Mit einer Prävalenz zwischen 1 und 2\%

\section{(2) Die Risiken der Kontrastmittelgabe müssen bei jedem Patienten gegen ihren Nutzen abgewogen werden}

Die Gefäßmedizin und -chirurgie ist im Wandel begriffen: Die im Vergleich zu den klassischen operativen Verfahren weniger invasiven interventionellen Behandlungstechniken werden deutlich zunehmen. In der Gefäßchirurgie lesen wir in jeder Ausgabe über die potenziellen Vorteile dieser neuen Verfahren, und ihr Stellenwert wird in Zukunft vermutlich nicht geringer werden. Für unsere Patienten bedeuten sie in der Regel ein geringeres $\mathrm{Zu}$ gangstrauma, kürzere Verweildauern und oftmals ambulante Behandlungsmöglichkeiten bei Erkrankungen, die noch vor wenigen Jahren aufwendige Bypassoperationen und längere Krankenhausaufenthalte erforderlich gemacht haben. Die Kehrseite dieser Medaille sind aber Kompli- kationen, mit denen wir uns bisher noch zu wenig befasst haben, wie die kontrastmittelinduzierte Nephropathie. Es ist daher das erklärte Ziel dieses Schwerpunktheftes Kontrastmittelsicherheit, Ihnen als Leser Beiträge zu präsentieren, die sich mit Kontrastmittelnebenwirkungen und Strategien zu deren Vermeidung befassen, die von ausgewiesenen Experten auf diesem Gebiet erstellt worden sind.

Herzlichst, Ihre

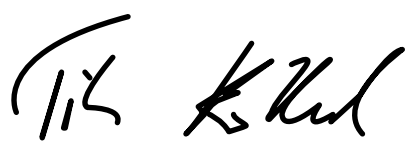

T. Kölbel

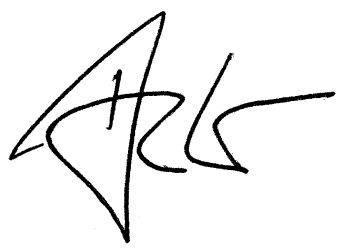

E.S. Debus

Korrespondenzadresse

Dr. T. Kölbel

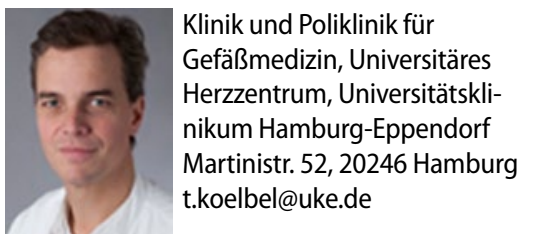

